\title{
The Proapoptotic Effect of Formononetin in Human Osteosarcoma Cells: Involvement of Inactivation of ERK and Akt Pathways
}

\author{
Yun Liu ${ }^{a}$ JinJie He $\mathrm{H}^{\mathrm{b}} \quad$ XiaoMing Chen ${ }^{\mathrm{a}}$ Jian Li ${ }^{\mathrm{a}}$ MaoRong Shen ${ }^{\mathrm{b}}$ WenJun Yu${ }^{\mathrm{b}}$ \\ Yuan Yang ${ }^{b}$ ZengMing Xiao \\ aDivision of Spinal Surgery, 1st Affiliated Hospital of Guangxi Medical University, Nanning, ${ }^{b}$ Division of \\ the fourth Orthopaedics, Guangxi Orthopaedic Traumatology Hospital, Nanning, China
}

\author{
Key Words \\ Formononetin • Calycosin • Osteosarcoma • Apoptosis • MAPK •PI3K/Akt
}

\begin{abstract}
Background: Previous studies have shown that some phytoestrogens inhibits proliferation and induces apoptosis in estrogen-dependent cancers via estrogen receptor (ER)-mediated signaling pathway. In view of the expression of ER in human osteosarcoma cells, the purpose of this study is to investigate whether formononetin and calycosin, two of the major isoflavones in Radix astragali, could also elicit anti-tumor activity against osteosarcoma, along with the underlying mechanism. Methods: Human osteosarcoma cells U2OS were respectively treated with various concentrations of formononetin or calycosin. Cell proliferation was determined by MTT assay, while apoptosis by flow cytometry. Next, the expression levels of apoptosisrelated genes ERK, Akt, BCl-2, Bax and caspase-3 were quantified by real-time PCR and Western blotting. Results: Formononetin exhibited higher anti-proliferative activities toward human osteosarcoma cells U2OS, when compared with calycosin. Therefore, U2OS cells were then respectively treated with various concentrations of formononetin, in order to elucidate the isoflavones-related signaling pathway. It was found that formononetin dose-dependently triggered apoptosis of U2OS cells in vitro. Furthermore, treatment of formononetin led to significant inactivation of ERK and Akt, followed by downregulation of $\mathrm{BCl}-2$, upregulation of Bax and finally increased expression of caspase-3. Conclusion: Formononetin is more effective than calycosin at promoting cell death of U2OS cells by induction of apoptosis, which is mediated by inactivation of ERK and Akt signaling pathways. Thus isoflavones, especially formononetin, may be useful as anti-cancer drugs for osteosarcoma through their apoptosisinducing effects.

Y. Liu and J. He contributed equally to this paper. 
Liu et al.: Proapoptotic Effect of Formononetin by Inactivation of ERK and Akt

\section{Introduction}

Osteosarcoma (OS) is regarded as the most common malignant bone tumor in children and adolescents, with a peak incidence in the second decade of life [1]. Due to the application of adjuvant chemotherapy after surgery, the overall survival rate for patients with nonmetastatic OS can be expected to be around $70 \%$ [2]. However, it falls to approximately $25 \%$ to $50 \%$ for patients with lung or bone metastases. And these survival rates appear to reach a plateau phase for the past 20 years. Furthermore, in some cases, the unwanted side effects of chemotherapy may result in treatment interruption or dose reduction, even in relatively young patients [3]. Therefore, there is an imperative to develop more effective drugs against OS.

Estrogens are known to have multiple functions in growth, differentiation and maintenance of many tissues, including bone containing estrogen receptor alpha (ER $\alpha$ ) and beta (ER $\beta$ ) [4]. Given the similar molecular structures between estrogens and phytoestrogens, it is possible that plant-derived phytoestrogens can mimic the effects of estrogen in ERpositive cells via binding to ERs [5]. The existence of ER $\beta$ in osteosarcoma has been reported by Dohi and colleagues, who examined the existence of ER in 28 cases of osteosarcoma using immunohistochemistry and observed ER $\beta$ expression in 23 cases [6]. Meanwhile, Chrzan et al reported that genistein, phytoestrogen from soybeans, elicits estrogenic responses in human osteosarcoma cell line G-292 through transactivation of ER $\beta 1$, indicating the estrogen-like effects of phytoestrogens in osteosarcoma [7]. Interestingly, there are several phytoestrogens that exhibit both estrogenic and antiestrogenic properties, thereby contributing to their anti-cancer properties on estrogen-dependent cancers. Phytoestrogens can be categorized as isoflavones, lignans, stilbenes and coumestans, which function much as natural selective estrogen receptor modulator [8]. The isoflavones formononetin and calycosin are two main active components of Radix astragali. As a traditional herbal medicine, Radix astragali has been widely used in China and East Asia for thousand years for the treatment of diabetes, hypertension, wound healing, cancers and so on [9] In previous studies, the formononetin and calycosin have been discussed to be anti-proliferative for breast and prostate cancers by promoting apoptosis and arresting cell cycle, which was obtained by upregulation of ER $\beta$ [10-12]. Accordingly, we speculate that formononetin and calycosin may also exert anticancer effects in ER-positive human osteosarcoma cells.

Targeting apoptotic pathways appears as promising approaches to prevent and treat cancers. Hence, the presentstudy aimed to investigate the biological activities of the isoflavones formononetin and calycosin in proliferation and apoptosis of human osteosarcoma cell line U20S, along with the underlying molecular mechanism. We here reported for the first time that formononetin suppressed cell growth by triggering apoptosis of U2-OS cells, which is mediated through inactivation of extracellular signal-regulated kinase (ERK) and serine/ threonine kinase (Akt) signaling pathways, including downregulation of B-cell lymphoma 2 (Bcl-2), upregulation of Bcl-2 associated protein X (Bax) and cleavage of caspase-3.

\section{Materials and Methods}

\section{Cell culture}

Experiments were performed on human osteosarcoma cell line U2OS obtained from the American Type Culture Collection (ATCC, Manassas, USA). Cells were grown at $37{ }^{\circ} \mathrm{C}$ and $5 \% \mathrm{CO}_{2}$ in Dulbecco's modified Eagle's medium (DMEM; Invitrogen, CA, USA), containing $10 \%$ fetal calf serum (FCS; Sigma-Aldrich, St. Louis, USA), $100 \mathrm{U} / \mathrm{ml}$ penicillin and $100 \mu \mathrm{g} / \mathrm{ml}$ streptomycin.

\section{MTT assay}

Cell proliferation was measured using 3-(4,5-dimethylthiazol-2-yl)-2,5-diphenyltetrrazoliumbromide (MTT) assay. U2OS cells $\left(3 \times 10^{3}\right.$ cells/well) were plated separately in a 96-well plate for $12 \mathrm{~h}$, and then treated with different concentrations of formononetin or calycosin $(0,5,10,20,30,40,60,80$ and 100 
$\mu M$ ). Formononetin $\left(\mathrm{C}_{16} \mathrm{H}_{12} \mathrm{O}_{4}\right)$ was purchased from Sigma-Aldrich (St. Louis, USA), and calycosin $\left(\mathrm{C}_{16} \mathrm{H}_{12} \mathrm{O}_{5}\right)$ was purchased from Phytomarker Ltd (Tianjin, China). Their stock solution was prepared by dissolving formononetin and calycosin in dimethyl sulfoxide (DMSO) and stored at $4{ }^{\circ} \mathrm{C}$ until use. After $48 \mathrm{~h}$, MTT was added into the U2OS cells to a final concentration of $0.5 \mathrm{mg} / \mathrm{ml}$ and incubated for $4 \mathrm{~h}$ at $37^{\circ} \mathrm{C}$. Next, the supernatant was discarded and $200 \mu \mathrm{L}$ DMSO was added to dissolve the formazan crystals. The optical density (OD) at $570 \mathrm{~nm}$ was determined with a spectrophotometer (Thermo Scientific, MA, USA).

Flow cytometry assay

After treatment with formononetin $(0,20,40$ and $80 \mu \mathrm{M})$ for $48 \mathrm{~h}$, U20S cells were harvested and rinsed with ice-cold PBS. Then cells were stained with Annexin V-fluorescein isothiocyanate (FITC) and propidium iodide (PI) (BD Biosciences, CA, USA) for $30 \mathrm{~min}$ at room temperature. Apoptotic ratio was detected by a FACS Aria flow cytometer (Beckman Coulter, FL, USA). Cells stained positive for Annexin V-FITC and negative for PI were considered early apoptotic.

Quantitative Real-time PCR ( $q R T-P C R)$ Assay

After treatment with formononetin $(0,20,40$ and $80 \mu \mathrm{M})$ for $48 \mathrm{~h}$, quantification of caspase-3 mRNA levels in U2OS cells was measured by qRT-PCR. Subsequent to isolation of total RNA with TRIzol Reagent (Invitrogen, CA, USA), the first strand cDNAs were generated with RevertAid TM First Strand cDNA Synthesis Kit (Fermentas, MD, USA) following the manufacturer's protocol. qRT-PCR was performed using a SYBR Green PCR kit (Roche, Mannheim, Germany) with GAPDH as an internal control.

Western blot assay

U2OS cells were incubated with formononetin at concentrations of $0,20,40$ and $80 \mu \mathrm{M}$ for $48 \mathrm{~h}$ or $80 \mu \mathrm{M}$ for $0,24,48,72$ and $96 \mathrm{~h}$. Then the protein samples were separated by sodium dodecyl sulfatepolyacrylamide gel electrophoresis (SDS-PAGE), transferred to polyvinylidene difluoride membrane (Millipore, MA, USA), and probed with primary antibodies against ERK (1:1000; Cell Signaling Technology, MA, USA), p-ERK (1:1000; Cell Signaling Technology, MA, USA), Akt (1:1000; Cell Signaling Technology, MA, USA), p-Akt (1:1000; Cell Signaling Technology, MA, USA), Bax (1:1000; Proteintech, IL, USA), Bcl-2 (1:1000; Proteintech, IL, USA) and GAPDH (1:10000; KangChen Bio-tech, Shanghai, China). Each band intensity was analyzed using the NIH Image J system (Bethesda, MD, USA) and normalized to the intensity of the loading controls.

Statistical analysis

All experiments were performed at least three times independently. Data are presented as mean \pm SD. Significant differences were assessed using One-way ANOVA tests. A p-value $<0.05$ was considered statistically significant.

\section{Results}

Formononetin and calycosin suppresses the proliferation in human osteosarcoma U2OS cells

The anti-proliferative effect of formononetin and calycosin on human osteosarcoma U2OS cells was respectively examined using MTT assay. As shown in Fig. 1, both formononetin and calycosin caused significant decrease in the proliferation of U2OS cells in a dose-responsive manner $(\mathrm{P}<0.05)$. Meanwhile, formononetin exerted stronger anti-proliferative effect on U20S cells when compared with calycosin. Given the close similarity between formononetin and calycosin, here formononetin that further enhanced inhibition of U2OS cell proliferation was applied to investigate the underlying mechanism of isoflavones in treating osteosarcoma.

Formononetin induces apoptosis in human osteosarcoma U2OS cells

To further investigate whether the growth inhibition induced by formononetin in human osteosarcoma cells was caused by apoptosis, U2OS cells were respectively treated with various concentrations of formononetin for $48 \mathrm{~h}$. Then the percentage of apoptotic 
Fig. 1. Antiproliferative effects of formononetin and calycosin on human osteosarcoma cell U2OS. Cells were treated with various concentrations of formononetin or calycosin for $48 \mathrm{~h}$, and then analyzed for viability by MTT assay. Data were shown as mean \pm SD. Results are representative of three independent experiments performed in triplicate. Compared with control group $(0 \mu \mathrm{M}){ }^{*} p<0.05$.

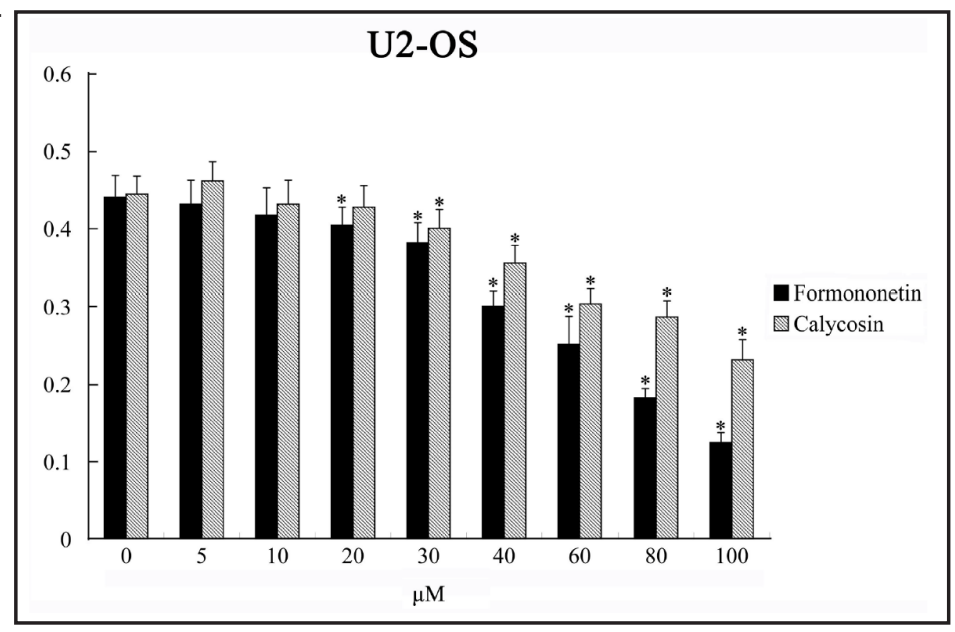

Fig. 2. Proapoptotic activity of formononetin detected by flow cytometry and qRT-PCR. After treatment of formononetin $(0,20,40$ and $80 \mu \mathrm{M})$, rates of early apoptotic U20S cells were measured by flow cytometric (A), while caspase-3 mRNA expression levels by qRTPCR (B). There are representative data from three independent experiments. Compared with control group $(0 \mu \mathrm{M}) * p<0.05$.

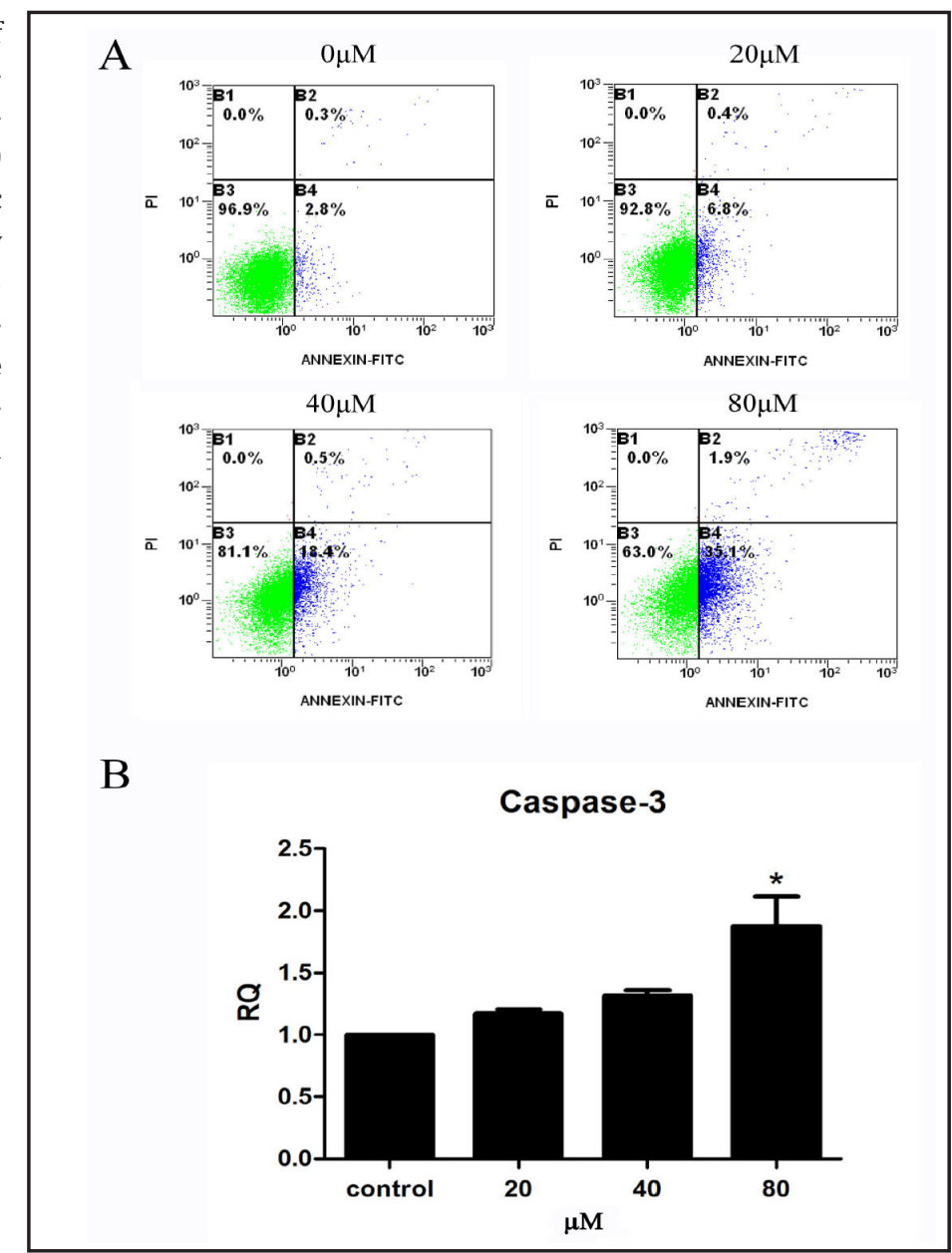

cells was measured by flow cytometry. It was found that the number of early apoptotic cells gradually increased from $0.4 \%(20 \mu \mathrm{M})$ to $35.1 \%(80 \mu \mathrm{M})$ that was statistically different from $0.5 \%$ in control group $(\mathrm{P}<0.05)$, as shown in Fig. 2A. Theses results suggested that growth suppression by formononetin in human osteosarcoma cells involved induction of apoptosis.

Activation of caspase-3 has been shown to initiate cleavage of a multitude of death substrates, resulting in cell apoptosis. To provide further evidence for formononetin-induced apoptosis, we examined caspase-3 activity in U2OS cells by qRT-PCR. Treatment with 
A
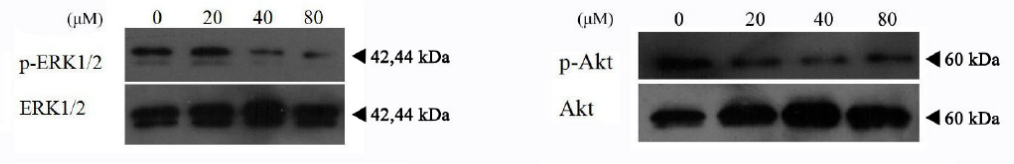

polyclonal-ERK1/2/total ERK1/2

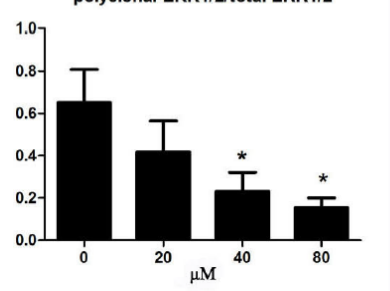

polyclonal-Akt/total Akt

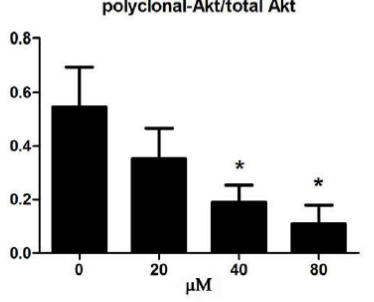

B
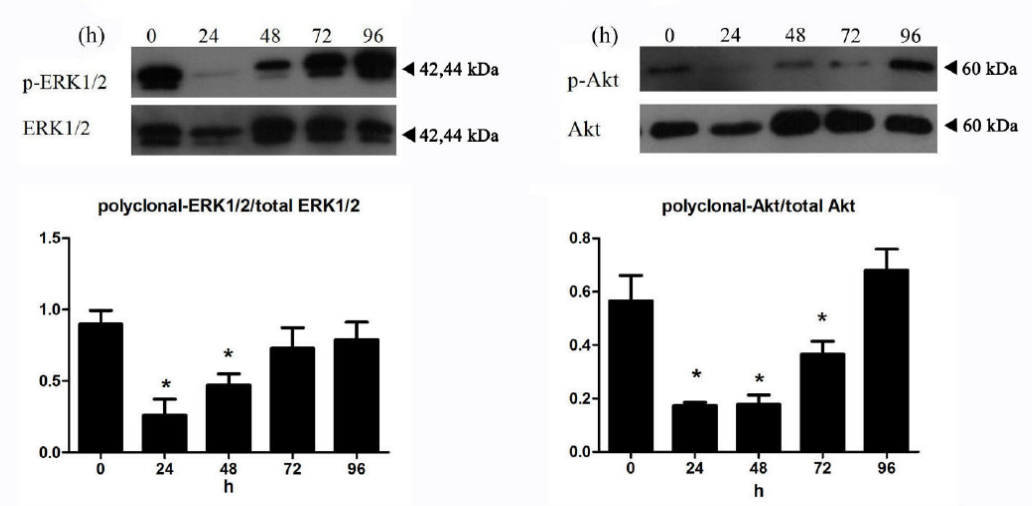

Fig. 3. Inhibited ERK and Akt proteins expression in human osteosarcoma cell U2OS by formononetin. Cells were treated with $0,20,40,80 \mu \mathrm{M}$ formononetin for $48 \mathrm{~h}(\mathrm{~A})$ or $80 \mu \mathrm{M}$ formononetin for $0,24,48,72,96 \mathrm{~h}$ (B), and then protein samples were labeled with specific antibodies against ERK, p-ERK, Akt and p-Akt. The antibodies to total ERK and Akt served as loading controls. There are representative data from three independent experiments. Compared with control group $(0 \mu \mathrm{M}$ or $0 \mathrm{~h}){ }^{*} p<0.05$.

formononetin dose-dependently increased levels of caspase-3 mRNA expression, especially in the high concentration group ( $80 \mu \mathrm{M})$ (Fig. 2B). These data clearly indicated that activation of caspase-dependent apoptosis is a major mechanism by which formononetin suppressed the growth of human osteosarcoma cells.

Inactivation of ERK and Akt by formononetin in human osteosarcoma U2OS cells

It has been reported that activation of mitogen-activated protein kinase (MAPK) and Phosphatidylinositol 3-kinase (PI3K)/Akt pathways is associated with caspase-3 expression. Thus the activation of ERK and Akt in formononetin-treated U2OS cells were evaluated by Western blot, so as to determine whether the modulations of ERK and Akt signaling pathways account for apoptosis induced by formononetin. We found that the phosphorylation levels of both ERK and Akt were markedly decreased in a dose-dependent manner following exposure to formononetin $(\mathrm{P}<0.05)$, as shown in Fig. 3A. Next, U2OS cells were incubated with 80 $\mu \mathrm{M}$ formononetin for different periods. Unexpectedly, although formononetin reduced phosphorylation of ERK and Akt, both p-ERK and p-Akt expression increased over time (Fig. $3 \mathrm{~B}$ ), indicating that formononetin strongly regulates apoptosis-related gene expression in a relatively short term and reaches a maximal response at $24 \mathrm{~h}$. Hence, these results suggested that inactivation of ERK and AKT might contribute to formononetin-induced apoptosis. 
Fig. 4. Effect of formononetin on the expression of Bax and Bcl-2 proteins in human osteosarcoma cell U20S. U2OS cells were treated with 0,20 , 40 and $80 \mu \mathrm{M}$ formononetin for $48 \mathrm{~h}$. Then expression of Bax and Bcl-2 was determined by Western blot, with GAPDH served as loading controls. There are representative data from three independent experiments. Compared with control group $(0 \mu \mathrm{M})$ ${ }^{*} p<0.05$.

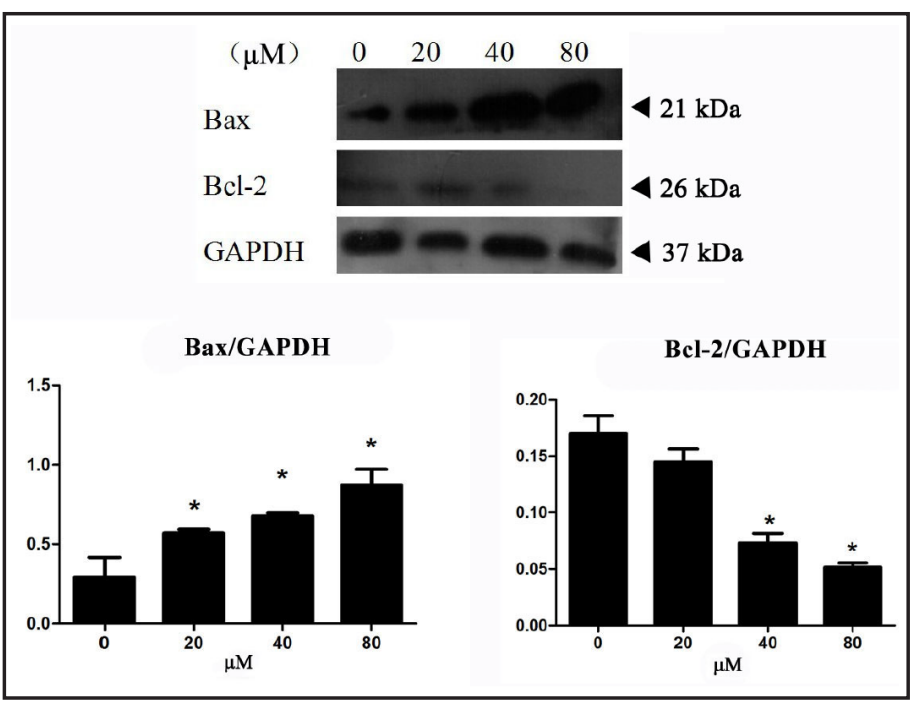

Formononetin inhibits Bcl-2 expression but enhances Bax expression in human osteosarcoma U2OS cells

As downstream targets of ERK and Akt signaling pathway, Bcl-2 family proteins are closely correlated with apoptosis, of which Bcl-2 is a cell death inhibitor and Bax has a critical role in promoting apoptosis. We next detected the expression of Bcl-2 and Bax proteins in U2OS cells after treatment with formononetin by Western blot. It was demonstrated that, accompanied by inhibition of ERK and Akt phosphorylation, Bcl-2 expression levels were dose-dependently decreased $(\mathrm{P}<0.05)$, whereas Bax expression was up-regulated $(\mathrm{P}<0.05)$, as shown in Fig. 4. It was identified that Bcl-2 and Bax might be downstream targets of ERK and Akt signaling pathways, which is involved in formononetin-triggered apoptosis in human osteosarcoma U2OS cells.

\section{Discussion}

It is known that osteosarcomas frequently occur in the second decade of life, when synthesis of sex steroids such as estrogen or androgen also reaches their peak, implying the involvement of sex steroids and receptors in the development of osteosarcomas [13]. Furthermore, the findings by Luo et al demonstrated that estrogen plays a key role in the proliferation of human osteosarcoma cells, and thus ER blockers could significantly suppress growth of human osteosarcomas [14, 15].

Recently, phytoestrogens have received considerable attention because of their ability to bind estrogen receptors. Meanwhile, accumulating evidence proves that some phytoestrogens exhibit antiestrogenic activity via ER-mediated signaling pathway [16-18]. For example, Nakamura et al reported that genistein, an isoflavone found in soy, inhibited proliferation and decreased invasive potential of human osteosarcoma cell line LM8, indicating the role of phytoestrogens in the prevention and treatment of osteosarcomas [19]. Formononetin and calycosin are two other main components of isoflavones that have been proved the potential anti-cancer properties. Previously, ER $\beta$-mediated proliferative inhibition and apoptosis by formononetin or calycosin has been reported in ER-positive human breast cancer cells MCF7 and T-47D, suggesting the potential use of isoflavones in ER $\beta$-positive cancer $[20,21]$. Considering the observed ER $\beta$ expression in human osteosarcomas, we here firstly explored the inhibitory effect of formononetin and calycosin on human osteosarcoma cell line U2OS in vitro. As expected, both formononetin and calycosin markedly inhibited cell proliferation of U2OS cells in concentration-dependent manner. Moreover, the growth inhibitory effects induced by formononetin were greater than calycosin, which indicates that formononetin 
may be superior to calycosin in treating human osteosarcoma. Accordingly, we next chose formononetin to explore how isoflavones mediate anti-proliferation effects on human osteosarcoma U2OS cells. Furthermore, it was shown that apoptosis is the predominant mode of formononetin-mediated cell death, as determined by increased early apoptotic rate with flow cytometry measurement.

Caspases family participates in several different pathways, including inflammatory, development and apoptotic pathways [22]. By cleaving and activating each other, caspases family assures proper execution of cell death. Caspase- 3 is the best known apoptotic executor that is responsible for induction of apoptosis program through activation of caspase-activated DNase and subsequent cleavage of DNA [23]. Previous studies have shown that formononetin-induced death of cancer cells is dependent on caspase-3 activation [24]. Similarly, here treatment of formononetin resulted in upregulation of caspase- 3 in U2OS cells, further confirming that this anti-proliferative activity is mediated by caspase-dependent cell apoptosis.

Next, we tried to identify the upstream signaling of caspase-3 in formononetin-treated human osteosarcoma cell U2OS. It is suggested that activation of MAPK signaling pathway is involved in the promotion of cancer cell apoptosis by formononetin $[10,20]$. Thus, we explored whether similar mechanisms apply to formononetin-induced cytotoxicity in human U2OS cells. MAPKs consist of three major subfamilies in mammalian cells, including p38 MAPK, ERK and c-Jun N-terminal kinase (JNK), among which the reduced ERK activation results in caspase-3-dependent apoptosis [25]. Then we here demonstrated that formononetin markedly decreased the phosphorylation of ERK in human osteosarcoma cells. Moreover, in present study, it was found that reduced ERK phosphorylation was accompanied by inactivation of Akt. PI3K/Akt signaling pathway is a pro-survival signals activated by growth factors, cytokines and hormones [26]. Activation of Akt favors cell survival through the regulation of apoptotic proteins, including Bcl-2 family and caspases $[27,28]$. Thus we believe that, besides inactivation of ERK, the observed downregulation of Akt may be also involved in formononetin-induced apoptosis. These results suggested that formononetin induced caspase-dependent apoptosis in human osteosarcoma cells through both ERK and Akt signaling.

To further investigate how formononetin-mediated inactivation of ERK and Akt induces caspase- 3 activation, we then detected the alteration in expression of Bcl-2 family members. Bcl-2 family could regulate the intrinsic mitochondrial pathway of apoptosis mainly by controlling cytochrome $C$ release, which has been identified as upstream regulator of caspases cascade $[29,30]$. Bcl-2 family comprises antiapoptotic components (e.g. Bcl-2 and Bcl-xL) and proapoptotic components (e.g. Bax, Bak and Bad) [31]. Moreover, numerous studies have shown that PI3K/Akt or MAPK pathways could result in regulation of Bax and Bcl-2, leading to activation of caspase-3 and cell death [32-34]. In present study, in response to regulation of ERK and Akt, Bax expression was significantly increased and Bcl-2 expression was downregulated with treatment of formononetin, leading to apoptotic cell death.

Taken together, this study is the first report to demonstrate the inhibitory activity of formononetin and calycosin on human osteosarcoma cells in vitro. In addition, inactivation of ERK and Akt is required for isoflavones-induced caspase-dependent cell apoptosis. Our study could provide insights into potential therapeutic use of isoflavones in clinical treatment of osteosarcomas.

\section{Acknowledgements}

This research was supported by grants from the Youth Fund of Guangxi Medical University (NO. GXMUYSF201326). 
Liu et al.: Proapoptotic Effect of Formononetin by Inactivation of ERK and Akt

\section{Reference}

1 Zhu Y, Zhou J, Ji Y, Yu B: Elevated expression of AKT2 correlates with disease severity and poor prognosis in human osteosarcoma. Mol Med Rep 2014;10:737-742.

2 Arndt CA, Rose PS, Folpe AL, Laack NN: Common musculoskeletal tumors of childhood and adolescence. Mayo Clin Proc 2012;87:475-487.

- 3 Robert G, Chappé C, Taque S, Bruneau B, Gandemer V: Hearing loss during osteosarcoma chemotherapy: when acute ifosfamide toxicity revealed unnoticed methotrexate. J Pediatr Hematol Oncol 2014;36:e100102.

-4 Manolagas SC, O'Brien CA, Almeida M: The role of estrogen and androgen receptors in bone health and disease. Nat Rev Endocrinol 2013;9:699-712.

5 May FE: Novel drugs that target the estrogen-related receptor alpha: their therapeutic potential in breast cancer. Cancer Manag Res 2014;6:225-252.

6 Svoboda M, Hamilton G, Thalhammer T: Steroid hormone metabolizing enzymes in benign and malignant human bone tumors. Expert Opin Drug Metab Toxicol 2010;6:427-437.

7 Rietjens IM, Sotoca AM, Vervoort J, Louisse J: Mechanisms underlying the dualistic mode of action of major soy isoflavones in relation to cell proliferation and cancer risks. Mol Nutr Food Res 2013;57:100-113.

-8 Lin AH, Li RW, Ho EY, Leung GP, Leung SW, Vanhoutte PM, Man RY: Differential ligand binding affinities of human estrogen receptor- $\alpha$ isoforms. PLoS One 2013;8:e63199.

-9 Yang Y, Chin A, Zhang L, Lu J, Wong RW: The role of traditional Chinese medicines in osteogenesis and angiogenesis. Phytother Res 2014;28:1-8.

10 Zhang X, Bi L, Ye Y, Chen J: Formononetin induces apoptosis in PC-3 prostate cancer cells through enhancing the Bax/Bcl-2 ratios and regulating the p38/Akt pathway. Nutr Cancer 2014;66:656-661.

11 Chen J, Zhao X, Ye Y, Wang Y, Tian J: Estrogen receptor beta-mediated proliferative inhibition and apoptosis in human breast cancer by calycosin and formononetin. Cell Physiol Biochem 2013;32:1790-1797.

-12 Huang WJ, Bi LY, Li ZZ, Zhang X, Ye Y: Formononetin induces the mitochondrial apoptosis pathway in prostate cancer cells via downregulation of the IGF-1/IGF-1R signaling pathway. Pharm Biol 2014;52:466470.

13 Botter SM, Neri D, Fuchs B: Recent advances in osteosarcoma. Curr Opin Pharmacol 2014;16C:15-23.

14 Zhang C, Peng J, Wu S, Jin Y, Xia F, Wang C, Liu K, Sun H, Liu M: Dioscin promotes osteoblastic proliferation and differentiation via Lrp5 and ER pathway in mouse and human osteoblast-like cell lines. J Biomed Sci 2014;21:30.

15 Lee CH, Huang YL, Liao JF, Chiou WF: Ugonin K-stimulated osteogenesis involves estrogen receptordependent activation of non-classical Src signaling pathway and classical pathway. Eur J Pharmacol 2012;676:26-33.

-16 Hwang KA, Park MA, Kang NH, Yi BR, Hyun SH, Jeung EB, Choi KC: Anticancer effect of genistein on BG-1 ovarian cancer growth induced by $17 \beta$-estradiol or bisphenol A via the suppression of the crosstalk between estrogen receptor $\alpha$ and insulin-like growth factor-1 receptor signaling pathways. Toxicol Appl Pharmacol 2013;272:637-646.

17 Thelen P, Wuttke W, Seidlová-Wuttke D: Phytoestrogens selective for the estrogen receptor beta exert antiandrogenic effects in castration resistant prostate cancer. J Steroid Biochem Mol Biol 2014;139:290-293.

18 Collins-Burow BM, Antoon JW, Frigo DE, Elliott S, Weldon CB, Boue SM, Beckman BS, Curiel TJ, Alam J, McLachlan JA, Burow ME: Antiestrogenic activity of flavonoid phytochemicals mediated via the c-Jun N-terminal protein kinase pathway. Cell-type specific regulation of estrogen receptor alpha. J Steroid Biochem Mol Biol 2012;132:186-193.

19 Nakamura A, Aizawa J, Sakayama K, Kidani T, Takata T, Norimatsu Y, Miura H, Masuno H: Genistein inhibits cell invasion and motility by inducing cell differentiation in murine osteosarcoma cell line LM8. BMC Cell Biol 2012;13:24.

20 Chen J, Sun L: Formononetin-induced apoptosis by activation of Ras/p38 mitogen-activated protein kinase in estrogen receptor-positive human breast cancer cells. Horm Metab Res 2012;44:943-948.

21 Tian J, Duan YX, Bei CY, Chen J: Calycosin induces apoptosis by upregulation of RASD1 in human breast cancer cells MCF-7. Horm Metab Res 2013;45:593-598.

22 Hensley P, Mishra M, Kyprianou N: Targeting caspases in cancer therapeutics. Biol Chem 2013;394: 831-843. 
23 Fiandalo MV, Kyprianou N: Caspase control: protagonists of cancer cell apoptosis. Exp Oncol 2012;34:165175.

24 Mansoor TA, Ramalho RM, Luo X, Ramalhete C, Rodrigues CM, Ferreira MJ: Isoflavones as apoptosis inducers in human hepatoma HuH-7 cells. Phytother Res 2011;25:1819-1824.

25 Diab S, Kumarasiri M, Yu M, Teo T, Proud C, Milne R, Wang S: MAP kinase-interacting kinases-emerging targets against cancer. Chem Biol 2014;21:441-452.

-26 Dupont J, Reverchon M, Cloix L, Froment P, Ramé C: Involvement of adipokines, AMPK, PI3K and the PPAR signaling pathways in ovarian follicle development and cancer. Int J Dev Biol 2012;56:959-967.

-27 Shin DY, Kim GY, Hwang HJ, Kim WJ, Choi YH: Diallyl trisulfide-induced apoptosis of bladder cancer cells is caspase-dependent and regulated by PI3K/Akt and JNK pathways. Environ Toxicol Pharmacol 2014;37:7483.

28 Rahmani M, Aust MM, Attkisson E, Williams DC Jr, Ferreira-Gonzalez A, Grant S: Dual inhibition of Bcl-2 and Bcl-xL strikingly enhances PI3K inhibition-induced apoptosis in human myeloid leukemia cells through a GSK3- and Bim-dependent mechanism. Cancer Res 2013;73:1340-1351.

29 Monian P, Jiang X: Clearing the final hurdles to mitochondrial apoptosis: regulation post cytochrome C release. Exp Oncol 2012;34:185-191.

30 Martinou JC, Youle RJ: Mitochondria in apoptosis: Bcl-2 family members and mitochondrial dynamics. Dev Cell 2011;21:92-101.

-31 Soriano ME, Scorrano L: The interplay between BCL-2 family proteins and mitochondrial morphology in the regulation of apoptosis. Adv Exp Med Biol 2010;687:97-114.

32 Park JS, Kwon JK, Kim HR, Kim HJ, Kim BS, Jung JY: Farnesol induces apoptosis of DU145 prostate cancer cells through the PI3K/Akt and MAPK pathways. Int J Mol Med 2014;33:1169-1176.

-33 Vitagliano O, Addeo R, D'Angelo V, Indolfi C, Indolfi P, Casale F: The Bcl-2/Bax and Ras/Raf/MEK/ERK signaling pathways: implications in pediatric leukemia pathogenesis and new prospects for therapeutic approaches. Expert Rev Hematol 2013;6:587-597.

34 Miwa S, Sugimoto N, Yamamoto N, Shirai T, Nishida H, Hayashi K, Kimura H, Takeuchi A, Igarashi K, Yachie A, Tsuchiya H: Caffeine induces apoptosis of osteosarcoma cells by inhibiting AKT/mTOR/S6K, NF-кB and MAPK pathways. Anticancer Res 2012;32:3643-3649. 